\title{
Mortalidade segundo causas: considerações sobre a fidedignidade dos dados
}

\author{
Ruy Laurenti, ${ }^{1}$ M. Helena P. de Mello Jorge ${ }^{1}$ e Sabina L. D. Gotlieb ${ }^{1}$
}

Como citar Laurenti R, Mello Jorge MHP, Gotlieb SLD. Mortalidade segundo causas: considerações sobre a fidedignidade dos dados. Rev Panam Salud Publica. 2008;23(5):349-56.

RESUMO As estatísticas de mortalidade são usadas em epidemiologia e saúde pública como indicador de nível de saúde, em avaliações de programas de saúde e em estudos populacionais visando a comparar tendências temporais e diferenças geográficas. Uma das variáveis utilizadas nesse tipo de análise é a causa básica da morte. Entretanto, existem críticas quanto à qualidade das estatísticas baseadas nas causas de morte declaradas pelos médicos nos atestados de óbito. $O$ objetivo deste artigo é refletir sobre a fidedignidade das causas de morte declaradas pelos médicos nos atestados de óbito, com base em estudos realizados segundo diferentes metodologias, e comentar a validade das estatísticas de mortalidade segundo causas.

Palavras-chave Coeficiente de mortalidade, análise estatística, atestado de óbito.

Há quase uma década, Cook (1) comentou que desde 1837 os atestados de óbito vêm sendo utilizados para produzir estatísticas de mortalidade na Inglaterra. Atualmente, mais de 160 anos depois, as estatísticas de mortalidade continuam sendo a mais importante fonte de informação disponível sobre a saúde da população. Elas têm sido usadas para avaliar desigualdades sociais, tendências temporais e diferenças regionais e ocupacionais. Têm também sido utilizadas no planejamento de serviços de saúde e na pesquisa.

\footnotetext{
1 Universidade de São Paulo (USP), Faculdade de Saúde Pública, Departamento de Epidemiologia. Enviar correspondência a Ruy Laurenti no seguinte endereço: Avenida Dr. Arnaldo 715, CEP 01246-904, São Paulo, SP, Brasil. E-mail: laurenti@ usp.br
}

A Inglaterra também é considerada o berço das estatísticas de mortalidade segundo causas, pois é daquele país a primeira publicação sobre essas estatísticas, que incluía, entre outras variáveis, a causa da morte (2). O modo tradicional de elaborar as estatísticas de mortalidade por causas envolve um sistema no qual as informações relativas a cada uma das mortes ocorridas são coletadas sempre com base no atestado de óbito, que contém, entre outras variáveis, a causa de morte preenchida pelo médico. Nesse sistema, adota-se uma definição internacional clara e bastante precisa sobre a causa a ser escolhida como a causa da morte, dentre as várias declaradas pelo médico. É a chamada causa básica da morte, definida na Décima Revisão da Classificação Internacional de Doenças e Problemas Relacionados à Saúde
(CID-10) como “(a) a doença ou lesão que iniciou a cadeia de acontecimentos patológicos que conduziram diretamente à morte ou (b) as circunstâncias do acidente ou violência que produziram a lesão fatal".

John Graunt, sempre citado em livros de epidemiologia e de estatísticas de saúde e considerado o "pai" da epidemiologia quantitativa, das estatísticas de mortalidade e da classificação de doenças, já manifestava preocupação quanto à exatidão dos dados de mortalidade. Apresentou, entre outras, uma descrição pormenorizada das listas de mortalidade (bills of mortality), incluindo aspectos relativos à qualidade dos dados. Nordenfelt (3), comentando as causas de morte pela lista criada por Graunt, citou que este estava consciente da existência de possíveis erros; porém, seu trabalho reve- 
lava uma grande perspicácia nesse aspecto. Rothman (4) descreveu os comentários de Graunt sobre a exatidão dos dados de mortalidade, particularmente quanto à dificuldade de se declarar a verdadeira causa de morte.

Tomando em consideração todos esses aspectos, o objetivo do presente artigo é refletir sobre a fidedignidade das causas de morte declaradas pelos médicos nos atestados de óbito, com base em estudos realizados segundo diferentes metodologias. Visa também a comentar a validade das estatísticas de mortalidade segundo causas. Não se pretende apresentar qualquer metodologia nova, mas, tão somente, comentar os diferentes tipos de estudos que vêm sendo utilizados para avaliar a fidedignidade dos dados referentes às causas de morte.

\section{FIDEDIGNIDADE DA CAUSA DE MORTE DECLARADA PELOS MÉDICOS NO ATESTADO DE ÓBITO}

O termo "causa de morte" refere-se, sempre, à causa básica, definida pela Organização Mundial da Saúde (OMS) e incluída nas sucessivas revisões da CID. A partir da CID-6 foi adotado, também, o modelo internacional para a declaração da causa da morte. Esse modelo passou a ser usado por todos os países membros e, juntamente com a definição da causa básica, nele anotada, possibilitou a comparabilidade das estatísticas de mortalidade por causas em séries geográficas e históricas.

Várias são as maneiras de avaliar a acurácia das causas de morte informadas nas estatísticas de mortalidade. Esquematicamente, os estudos com foco nessa questão podem ser classificados, segundo os autores deste trabalho, em cinco categorias: 1) estudos que comparam as informações do atestado de óbito com os achados de autopsia; 2) estudos que comparam as informações do atestado de óbito com os dados clínicos; 3) estudos que comparam as informações do atestado de óbito com dados clínicos e de entrevistas com familiares do falecido; 4) estudos que avaliam as diferenças entre codificadores na classifica- ção da causa básica da morte e 5) outros tipos de estudos.

\section{Comparação entre as informações do atestado de óbito e achados de autopsia}

Independentemente da avaliação das estatísticas de mortalidade por causas, sempre existiu o interesse em comparar diagnósticos clínicos aos resultantes do exame cadavérico. Um antigo estudo, muito citado, feito em 1900 , por Cabot, comparou os resultados de 3000 autopsias com os respectivos dados clínicos (5). A concordância entre esses diagnósticos variou para as diferentes doenças (de 16 a 95\%). O autor comenta, ainda, que as doenças que levavam rapidamente à morte tinham um período pequeno de observação e que a história clínica nem sempre oferecia elementos para um diagnóstico bem feito; dessa maneira, havia baixa concordância. É preciso lembrar que a pesquisa foi feita em 1900 e que, atualmente, técnicas novas, mais avançadas, possibilitam um diagnóstico correto, na maioria das vezes em pouco tempo.

Wells, na segunda década do século $\mathrm{XX}$, comparou os diagnósticos de 3712 autopsias com dados clínicos e encontrou um erro de $36,5 \%$ nos diagnósticos clínicos dos casos de neoplasias. Isso levou o autor a concluir que as estatísticas vitais eram falhas para câncer (6).

Outra investigação muito citada é a de Swartout e Webster (7), com 8080 casos do Los Angeles General Hospital, na Califórnia, Estados Unidos. Foi relatada uma concordância média de 79 a $89 \%$ para os casos de câncer, dependendo do local e da morfologia do tumor diagnosticado. Pesquisas desse tipo são relativamente freqüentes em câncer. São exemplos os trabalhos de Bauer e Robbins (8), Erlich et al. (9), Gobbato et al. (10) e Ron et al. (11), que avaliaram a concordância dos diagnósticos nos atestados de óbito com os obtidos pelas autopsias entre sobreviventes de bombardeio atômico.

Recentemente, Sanches comparou o preenchimento dos atestados de óbito com os resultados de autopsias em um hospital universitário de São Paulo, Brasil (12). A autora comparou, em 258 óbitos, a história clínica com as autopsias, a história clínica com o atestado de óbito e o atestado de óbito com a autopsia. Para o conjunto (três tipos de comparações), houve $45(17,5 \%)$ casos discordantes, sendo $28(62 \%)$ entre o atestado e a autopsia. Em 17 (38\%) casos houve concordância entre o atestado de óbito e a autopsia, mas discordância entre a história clínica e a autopsia.

A maioria dos pesquisadores que discutem esse tema assume que os achados de autopsia constituem-se na base para avaliar a acurácia da informação no atestado de óbito, sendo considerados como o padrão-ouro. Ron et al. (11) apontam que alguns dados clínicos podem ser importantes na determinação da causa da morte, mas não são considerados pelo patologista que faz a autopsia, geralmente por desconhecimento.

É preciso salientar que mesmo que fossem feitas autopsias em 100\% das mortes, ainda assim não existiria exatidão na informação da causa básica, porque nem todos os patologistas sabem preencher corretamente os atestados em relação às causas, conforme as diretrizes da OMS. Esse fato já foi constatado pelos autores deste artigo em várias investigações sobre mortalidade, nas quais observaram casos com autopsias bem feitas em cujos atestados de óbito constavam, somente, "edema pulmonar", "broncopneumonia", "insuficiência cardíaca", entre outros. Não raro, também, houve casos onde aparecia como causa uma neoplasia maligna que era, tão somente, um achado de autopsia, sem qualquer manifestação clínica relacionada à morte.

\section{Comparação entre as informações do atestado de óbito e os dados clínicos}

Neste caso, a exatidão da informação sobre causas no atestado de óbito é avaliada pela comparação com as informações clínicas obtidas de 
prontuários médicos ou por meio de entrevistas com os médicos que cuidaram do paciente que morreu. Esta talvez seja a maneira mais freqüente de avaliar a acurácia da declaração das causas de morte.

Um trabalho clássico bastante citado foi feito na Inglaterra por Alderson e Meade (13), que compararam os diagnósticos constantes do prontuário hospitalar com a declaração da causa da morte nos atestados, tendo sido detectada discordância de 39\%. O mesmo tipo de estudo foi realizado no Havaí, por Schwartz, que estimou discordância de 20\% (14). Na análise de 9724 casos de morte em hospitais de Vermont, nos Estados Unidos, Gittelsohn e Senning (15) observaram concordância de $72 \%$. Em outras pesquisas que utilizaram a mesma metodologia - na Venezuela (16), na Espanha (17) e no Kuwait (18) - foram encontradas discordâncias.

No Brasil, o primeiro estudo deste tipo parece ter sido o de Laurenti (19), publicado em 1974. Nesse estudo, foi analisada uma amostra de óbitos gerais ocorridos no Município de São Paulo, em 1972 e 1973. Os atestados foram comparados com os dados registrados nos prontuários médicos de hospitais de todos os tipos, tendo sido encontrada concordância de 62,3\%. Pouco mais de 10 anos depois, o mesmo autor e colaboradores compararam os atestados de óbito com os diagnósticos existentes nos prontuários médico-hospitalares de mulheres de 10 a 49 anos residentes no Município de São Paulo que haviam morrido. $\mathrm{O}$ enfoque principal foi avaliar a exatidão da declaração das mortes por causas maternas. Os autores verificaram que o número real de mortes por essas causas era $124 \%$ maior do que o obtido pelo atestado de óbito original (20). Nas décadas de 1980 e 1990, vários estudos similares foram conduzidos no Brasil, entre outros, os de Carvalho et al. (21), Niobey et al. (22), Nobre et al. (23), Schnitman (24) e Monteiro (25).

Da mesma maneira, como já comentado, no caso da comparação com os dados clínicos de prontuários médicohospitalares, a experiência dos autores deste resgate histórico tem mostrado que existem, muitas vezes, excelentes prontuários, mas que a declaração do médico no atestado de óbito nem sempre corresponde aos diagnósticos neles registrados. Não raro aparecem, apenas, "parada cardíaca", "insuficiência cardíaca" e, muito freqüentemente, "septicemia", sem informar qual doença ou lesão iniciou a sucessão de eventos, isto é, a causa básica que deveria aparecer nas estatísticas de mortalidade por causas.

\section{Comparação entre o atestado de óbito e os dados clínicos e de entrevistas familiares}

Este tipo de avaliação é semelhante ao anterior; entretanto, além da coleta de dados clínicos, são feitas entrevistas no domicílio da pessoa falecida, utilizando o método chamado "autopsia verbal". Neste método, às questões sobre composição familiar são acrescidas perguntas sobre ocupação e hábitos como fumo e ingestão de bebidas alcoólicas, entre outros, e a família relata, na medida do possível, a história da doença da pessoa que faleceu.

Em nossa experiência, a entrevista familiar, de uma maneira geral, muito tem contribuído para a elucidação diagnóstica, particularmente nos casos em que o prontuário médico-hospitalar não é de boa qualidade ou contém apenas dados sobre o episódio terminal da doença que levou à morte, sem referência à doença inicial. Este tipo de avaliação é conhecido como "metodologia Puffer", sendo que, no caso de mortes de mulheres em idade fértil, a metodologia é conhecida como "RAMOS" (do inglês reproductive age mortality survey, ou seja, inquérito de mortalidade em idade reprodutiva).

Puffer e Verhoestraete (26), no estudo sobre mortalidade por doenças cardiovasculares, observaram diferenças marcantes entre países. Uma relevante questão, entre outras que surgiram, foi saber se as diferenças eram reais ou devidas a questões terminológicas ou até (principalmente) à qualidade do preenchimento dos atestados de óbito.
Para serem obtidos dados comparáveis, é necessário que as metodologias adotadas sejam uniformes, não somente na maneira de registrar as mortes, mas também quanto a declarar e classificar as causas. Realizou-se, assim, por proposta de Puffer, uma pesquisa internacional cujo objetivo principal foi proporcionar um quadro completo, o mais exato e comparável possível, sobre as causas de morte em adultos. Foram escolhidas 12 cidades (11 nas Américas e uma na Inglaterra) onde, durante 2 anos, foi estudada uma amostra de óbitos de adultos de 15 a 74 anos, utilizando a metodologia Puffer. No Brasil, foram incluídas as cidades de São Paulo e Ribeirão Preto; o período de referência foi 1962 a 1964 (27). Quanto à avaliação da fidedignidade da causa médica da morte, nas declarações, os resultados mostraram que ela deixava a desejar, particularmente quanto a causas específicas. Entretanto, quando se compararam grandes grupos de causas, como os capítulos da CID, não existiam grandes diferenças entre as declarações originais e as finais, após a investigação. É importante mencionar que, ainda que esses resultados se refiram a uma investigação feita há mais de 40 anos, eles merecem ser citados, não só por seu valor histórico, mas, principalmente, porque servem de modelo para as investigações atuais.

Puffer idealizou ainda outra investigação, desta vez com óbitos de menores de 5 anos de idade, seguindo a mesma metodologia e abrangendo 25 áreas da América Latina, uma dos Estados Unidos e outra do Canadá, sendo o período de referência 1968 a 1970. Além de numerosos resultados de grande interesse para a epidemiologia e mesmo para a administração de serviços de saúde, avaliou-se também a qualidade da declaração médica das causas de morte, observando-se diferenças entre as causas "originais" e as "finais", após a investigação (28).

Um estudo mais recente, relativo à investigação de mortes de mulheres em idade fértil nas 26 capitais brasileiras e no Distrito Federal, abrangendo um total de 7332 casos ocorridos durante o primeiro semestre de 2002, 
com a utilização do método RAMOS, está tendo seus resultados publicados $(29,30)$. Na comparação dos casos de morte por causas maternas, o número de óbitos nos atestados "originais" e nos "finais", após a investigação, foi de 144 e 239 casos, respectivamente. No momento, está sendo analisada a exatidão dos demais diagnósticos declarados pelos médicos (30).

\section{Diferenças entre codificadores na classificação da causa básica da morte}

A seleção da causa básica da morte e a sua conseqüente codificação seguem regras, normas e orientações que devem ser usadas internacionalmente e estão apresentadas nas sucessivas revisões da CID. Ocorre que, nem sempre, os codificadores são bem treinados ou, não raro, têm "inconscientemente" preferências por alguma doença ou diagnóstico; nesses casos, os médicos podem preencher correta e adequadamente os atestados de óbito, porém, a má codificação vai afetar a fidedignidade das estatísticas. Há publicações internacionais que discutem esse tema, podendo-se citar Kelson e Forebrother (31) e Percy e Dolman (32).

O Centro Colaborador da OMS para a Família de Classificações em Português (CBCD), da Faculdade de Saúde Pública da Universidade de São Paulo (USP), fez, em 1984, uma avaliação da codificação da causa básica, dando um mesmo conjunto de atestados de óbito a um grupo de codificadores. Os resultados mostraram haver discrepâncias (33). É preciso comentar que mesmo codificadores excelentes, considerados padrãoouro, podem ter algumas divergências. Laurenti (34) mostrou os resultados da comparação entre dois codificadores padrão-ouro, um no Brasil e outro nos Estados Unidos, para um conjunto de 1837 atestados de óbito. A codificação foi baseada na CID-8, e revelou:

a) concordância nos quatro algarismos do código da doença em 1651 casos $(90,4 \%)$;

b) concordância nos três primeiros algarismos do código da doença em 35 casos $(1,9 \%)$; c) concordância nos dois primeiros algarismos do código, porém com as duas causas básicas selecionadas pertencendo ao mesmo agrupamento de doenças, em 18 casos $(1,0 \%)$;

d) discordância que afetou a causa básica e o seu agrupamento, sendo as duas causas básicas classificadas em grupos diferentes de doenças, em 123 casos $(6,7 \%)$.

Levando-se em conta que as estatísticas de mortalidade mostram as doenças classificadas por agrupamentos de causas, nesse estudo as concordâncias em apenas três ou até dois algarismos em nada afetaram os resultados finais apresentados pelos dois codificadores. Assim, pode-se considerar que houve concordância em $93,3 \%$ dos casos. O mais importante a salientar é que dois codificadores considerados de alto padrão classificaram as causas básicas do mesmo lote de atestados em grupos diferentes de doenças em 6,7\% dos casos. Esse nível de erro na codificação, na realidade, deve ser maior quando se considera a codificação feita por órgãos oficiais encarregados de classificar os atestados de óbito, pois tais órgãos nem sempre dispõem de pessoal suficientemente habilitado para tal tipo de atividade.

\section{Outros tipos de estudos}

Dadas as características específicas das causas externas para a avaliação da fidedignidade das suas informações nos atestados de óbito, há estudos especiais para as mesmas. Ocorre que, às vezes, os médicos legistas, responsáveis pelo preenchimento desses atestados, somente declaram a natureza da lesão, e não as circunstâncias do acidente ou da violência (causas básicas), conforme determina a OMS. O esclarecimento pode ser obtido por meio de consulta aos laudos elaborados pelos legistas ou então aos boletins de ocorrência policial. Um estudo de Mello Jorge (35) sobre acidentes e violência em São Paulo mostra o ganho de informação que essa metodologia proporciona. Merece ser ci- tada a atuação do Programa de Aprimoramento das Informações sobre Mortalidade (Pro-Aim) de São Paulo e de outras secretarias de saúde que incorporaram a investigação como rotina de serviço (36), resultando, assim, em um decréscimo sensível na freqüência de mortes por causas externas de intenção indeterminada (37).

Um tipo freqüente de avaliação da qualidade da informação nos atestados de óbito é a mensuração da proporção de mortes nas quais o diagnóstico foi uma causa mal definida (incluindo os casos sem assistência médica). No Brasil, está havendo um decréscimo lento e gradativo dessa proporção, que é, atualmente, igual a $12 \%$ (36). Esse declínio é fruto da complementação dos dados registrados nas declarações de óbito com aqueles resgatados das demais fontes.

O uso de regras internacionais para a seleção e a codificação da causa básica da morte pode interferir na acurácia da informação. Assim, há uma impressão generalizada, entre os que trabalham com estatísticas de mortalidade, de que a aplicação do princípio geral na seleção da causa básica, em atestados de óbito, seria um indicativo da sua "boa qualidade". O princípio é aplicado quando o médico declara de maneira correta e lógica a seqüência, a partir da causa básica, na parte I do atestado de óbito.

Os autores deste trabalho concordam, em parte, com essa impressão. De fato, há preenchimentos que possibilitam aplicar o princípio geral; porém, o médico pode declarar causas não existentes, ou, então, causas existentes que, contudo, não foram as causas reais da morte.

Testar essa afirmação somente é possível quando se faz uma investigação com as metodologias Puffer ou RAMOS. Assim, utilizando-se os atestados originais e os refeitos, após investigação, de mortes de mulheres em idade fértil (30), verificou-se que o princípio geral foi utilizado em $80,4 \%$ dos originais (apenas para óbitos por causas naturais). Entretanto, a pesquisa permitiu verificar que, em 10,1\% dos casos onde se aplicou o princípio geral, não havia exatidão quanto à 
causa básica declarada. Dessa forma, não é totalmente verdadeira a questão de que a aplicação do princípio geral é indicativa da boa fidedignidade da causa básica.

Vários estudos, ainda, poderiam ser citados, como os de relacionamento (linkage) entre o atestado de óbito e a declaração de nascido vivo, isto é, estudos que utilizam os sistemas de informações sobre mortalidade (SIM) e sobre nascidos vivos (SINASC). Comparando os dados maternos do nascido vivo, na declaração de nascido vivo (SINASC), e os dados do atestado de óbito de uma mulher em idade fértil (SIM), residente no mesmo local, poderia ser reconhecida a presença de uma morte materna. Os relacionamentos (linkages) entre o SIM e outros sistemas de informações em saúde também permitiriam avaliar a qualidade da informação sobre a causa de morte (38).

\section{COMENTÁRIOS}

As estatísticas de mortalidade são amplamente usadas em epidemiologia e em outras áreas da saúde pública como indicador para a avaliação de programas de saúde da população, em estudos retrospectivos e prospectivos, e também para descrever e comparar tendências temporais e diferenças geográficas quanto à freqüência de causas de morte, em um país, em suas regiões ou entre países.

Apesar de essas estatísticas serem tradicionais e, ainda hoje, rotineiramente utilizadas, há críticas quanto à fidedignidade da informação sobre a variável causa da morte, declarada pelos médicos nos atestados de óbito. Vários estudos visando à avaliação dessas estatísticas vêm sendo realizados, pode-se dizer, desde que John Graunt publicou pela primeira vez os seus dados de mortalidade em Londres, em 1662. Ele mesmo, em seus trabalhos, fez críticas à qualidade da declaração da causa da morte.

É importante salientar que há várias maneiras de definir a causa de morte, para diferentes propósitos ou finalidades. Assim, uma definição clínica diz respeito à manifestação terminal de uma doença primária, por exemplo, insuficiência cardíaca congestiva, no caso de cardiopatias, ou então a lesões (fraturas, queimaduras e outras) e não ao acidente ou à violência que as motivou. Ou seja: do ponto de vista clínico, o interesse é pelo motivo da procura por assistência médica. Para os patologistas, baseados em autopsias, não existe uma definição propriamente dita, mas, nos laudos, geralmente, colocam o "evento terminal" e a doença que levou a esse evento. Os legistas declaram as causas de morte como sendo a natureza de lesão, e não as circunstâncias do agravo responsável pela mesma.

Há outros interesses ou maneiras de serem obtidas informações sobre as causas de morte. Atualmente, há agências, serviços ou, mesmo, instituições acadêmicas que monitoram as mortes relacionadas a reações adversas a drogas e a certos procedimentos médicos, como cirurgias. Os registros de doenças específicas, por exemplo, de câncer, estão geralmente interessados em dados sobre as mortes, para estudos de sobrevida, independentemente das causas e complicações que levaram à morte.

$\mathrm{Na}$ introdução deste trabalho, foi apresentada a definição de causa básica da morte. É importante salientar que, do ponto de vista da prevenção da morte, é preciso interromper a cadeia de eventos a partir dela ou, principalmente, prevenir a causa inicial, isto é, a causa básica, o que está muito bem explicado na definição apresentada na CID-10 (39). Fica muito claro que as ações que previnem uma doença ou evento inicial vão prevenir não apenas a morte, mas, também, influir no período de duração da doença ou da incapacidade que a precede e, dessa maneira, contribuir para um ganho potencial de saúde da população. O desenho do atestado de óbito, com a parte relativa à declaração das causas, bem como as instruções para seu preenchimento, visam a identificar a causa básica para as finalidades da saúde pública. A existência das definições e do modelo de atestado de óbito para a declaração das causas e o fato de as regras para a seleção da causa básica serem de uso internacional possibilitam a comparabilidade.

Mesmo assim, as estatísticas de causas de morte não são, ainda, completamente exatas, o que leva a um certo descrédito. Esse fato, mas não ele exclusivamente, gerou esforços para que existissem estatísticas disponíveis sobre as doenças ou a morbidade na população, o que seria mais útil à saúde pública.

Moriyama, um dos maiores estatísticos de saúde da atualidade, falecido em 2006, abordou muito apropriadamente essa questão de morbidade versus mortalidade (40). Comenta que as estatísticas de mortalidade serviram muito bem aos propósitos da saúde pública quando o seu maior desafio era o controle de doenças infecciosas. Com o seu declínio, o interesse passou para as chamadas doenças crônicas, casos em que nem sempre é fácil determinar qual a causa básica, não só pelo médico, mas, também, pela aplicação das regras de seleção. Acresce ainda que doenças menos fatais, muitas vezes bastante freqüentes, não estão adequadamente presentes nas estatísticas de mortalidade. Surgiu, então, a idéia de que seriam as estatísticas de morbidade, e não as de mortalidade, que deveriam ocupar esse papel. Porém, por melhores que sejam as estatísticas de morbidade, as de mortalidade apresentam vantagens e continuam sendo amplamente utilizadas.

O aprimoramento da declaração das causas de mortes nos atestados de óbito é uma preocupação da OMS, principalmente, por meio de sua rede de centros colaboradores para a Família de Classificações de Uso em Saúde. Esses centros vêm estimulando a realização de estudos e análises das estatísticas de mortalidade por causa. Existe, inclusive, um grupo de discussão (Mortality Reference Group) para tratar dessas questões.

Como fazer para melhorar a qualidade da informação sobre as causas de morte? Não há dúvida de que, obviamente, o papel do médico é fundamental quanto ao correto preenchimento dos atestados de óbito; entretanto, é preciso ter em mente que mesmo os atestados adequadamente 
preenchidos podem vir a ser codificados erradamente e, dessa maneira, não ser selecionada a causa básica que o médico declarou. Portanto, não é unicamente uma questão do preenchimento pelo médico, mas, isso sim, é preciso ter bons codificadores. No Brasil, com a municipalização da saúde pela implantação do Sistema Único de Saúde (SUS), a seleção e a codificação da causa básica passaram a ser feitas em nível municipal, resultando na necessidade de treinar um grande número de codificadores. É fácil entender que, quase certamente, há codificadores ainda não devidamente preparados, além do fato de poder haver divergências mesmo entre bons ou ótimos codificadores.

Com o intuito de uniformizar a seleção da causa básica da morte foi criado, pelo CBCD, um sistema de seleção eletrônica da causa básica, que já vem sendo utilizado em várias áreas do País. Este procedimento vem contribuindo para o aprimoramento da qualidade da informação.

É preciso, ainda, comentar que a causa declarada pelo médico é apenas sua opinião e, mesmo conhecendo muito bem as normas do correto preenchimento do atestado, ela pode estar incorreta. Há estudos que avaliam o preenchimento de atestados por diferentes médicos para um mesmo conjunto ou lote de histórias clínicas. Alguns analisam casos com histórias clínicas de diagnósticos variados (41) e outros com diagnósticos específicos, como doença isquêmica do coração (42), doenças respiratórias (43) e diabetes (44). Os diversos trabalhos mostram que há discordâncias, variando entre 10 e 20\%; portanto, as opiniões dos médicos influem na declaração da causa. Ao se abordar o fator médico, não se pode esquecer dos modismos. É de se destacar que termos como parada cardíaca e insuficiência de múltiplos órgãos são muito utilizados.

A questão do traçado do atestado de óbito é, às vezes, apresentada, e alguns estudos do tipo piloto fizeram alterações no modelo para verificar se ocorria uma melhora da qualidade. Um, na Inglaterra, mostrou não ter havido melhora significativa na declaração da causa, e os médicos julgavam muito mais útil receber instruções sobre como preencher adequadamente o atestado de óbito do que as mudanças no mesmo (45). Esse comentário é importante e oportuno, pois, na experiência dos autores deste artigo, em primeiro lugar, os médicos precisam saber para que servem as estatísticas sobre causas de morte. Em geral, esse aspecto é ignorado pela maioria dos médicos, inclusive professores do ensino médico. Tal fato precisa ser explicitamente reconhecido pelos responsáveis pelas ementas nas faculdades de medicina e, conseqüentemente, o ensino do atestado de óbito (finalidade e preenchimento), das causas de morte, de definições e usos das estatísticas de mortalidade, deve ser introduzido obrigatoriamente nos currículos desses cursos.

No Brasil, dado o número crescente de faculdades de medicina, observa-se que essa temática passa ao largo. De alguns anos para cá, nos exames de seleção para programas de residência médica, tem sido colocada uma questão sobre o preenchimento do atestado de óbito. Nessa questão, é apresentada uma história clínica e os candidatos devem preencher o atestado correspondente.

A OMS, por intermédio de seus centros colaboradores, tem procurado estimular a elaboração de material didático sobre o assunto. No Brasil, uma publicação (46) aborda aspectos médicos, estatísticos e jurídicos e inclui um conjunto de histórias clínicas reais, mostrando como foi preenchido o respectivo atestado de óbito e como deveria ter sido, na forma correta. Essa publicação é distribuída gratuitamente para médicos e estudantes de medicina, em larga escala. O Conselho Federal de Medicina, a partir desse manual, editou um manuscrito condensado e pretende distribuí-lo a todos os médicos brasileiros.

Seria muito importante que, no Brasil, as secretarias municipais de saúde estabelecessem um sistema de vigilância sobre a qualidade da informação médica nos atestados de óbito. Nesse sistema, ênfase especial deveria ser dada aos diagnósticos mal-definidos ou aos bem definidos que não indicam a causa básica. $O$ contato com os médicos é um importante fator educativo.

O Ministério da Saúde do Brasil vem estimulando a criação de serviços de verificação de óbitos (SVO) como forma de melhorar a declaração da causa de morte, particularmente nos casos sem assistência médica ou nos casos com assistência, porém com diagnóstico mal definido. Tal fato, aliado à educação médica, no sentido do preenchimento adequado do atestado e à boa codificação das causas, será, com certeza, um fator positivo para o aprimoramento da informação sobre causas de morte.

Um comentário final precisa ser feito. A situação da não completa exatidão das declarações da causa de morte não é uma questão específica do Brasil e os numerosos estudos apresentados mostram isso. Os autores, por meio do CBCD, têm participado de grupos de trabalho da rede de centros da OMS bem como de reuniões internacionais em que essa temática está incluída; a preocupação é internacional e, em muitos países, sérios esforços têm sido adotados visando a melhorar a situação. O que o Brasil tem feito, por meio de ações do Ministério da Saúde, do CBCD e de numerosas secretarias de saúde, está dando bons resultados, e acredita-se que a situação possa melhorar.

Sabe-se que uma exatidão de 100\%, ou muito próxima disso, dificilmente será alcançada, em nosso País ou em outro. Mas, mesmo assim, as estatísticas de causas de morte continuarão a ser muito utilizadas e, nesse sentido, é de se ressaltar que dois dos Objetivos do Desenvolvimento do Milênio estão expressos em termos de mortalidade. Além disso, um dos três componentes do Índice de Desenvolvimento Humano é uma medida de mortalidade (47).

Concluindo, é possível afirmar que, embora não sendo totalmente fidedignas, as estatísticas de mortalidade por causa trazem relevante contribuição à caracterização do perfil epidemiológico de uma população. 


\section{REFERÊNCIAS}

1. Cook L. Foreword. Death certification and mortality statistics: an international perspective. Em: Coleman M, Aylin P. Studies on medical and population subjects $n^{\circ} 64$. Londres: National Statistics; 2000. Pp. 39-46.

2. Greenwood N. Medical statistics from Graunt to Farr. Cambridge: University Press; 1948.

3. Nordenfelt L. Causes of death: a philosophical essay. Swedish council for planning and coordination of research. Uppsalla: Committee for Future Oriented Research; 1983.

4. Rothman KJ. Lessons from John Graunt. Lancet. 1996;347(8993):37-9.

5. Cabot RC. Diagnostic pitfalls identified during a study of three thousand autopsies. JAMA. 1912;59:2295-8.

6. Wells HG. Relation of clinical to necropsy diagnosis in cancer and value of existing cancer statistics. JAMA. 1923;80:737-40.

7. Swartout HO, Webster RG. To what degree are mortality statistics dependable? Am J Public Health Nations Health. 1940;30(7): 811-5.

8. Bauer FW, Robbins SL. An autopsy study of cancer patients. 1. Accuracy of the clinical diagnosis (1955 to 1965). JAMA. 1972;221:1471-4.

9. Erlich D, Li-Sik M, Modan B. Some factors affecting the accuracy of cancer diagnosis. J Chronic Dis. 1975;28(7-8):359-64.

10. Gobbato F, Vecchiet F, Barbierato D, Melato M, Manconi R. Inaccuracy of death certificate diagnoses in malignancy: an analysis of 1,405 autopsied cases. Hum Pathol. 1982;13(11): 1036-8.

11. Ron E, Carter R, Jablon S, Mabuchi K. Agreement between death certificates and autopsy diagnoses among atomic bomb survivors. Epidemiology. 1994;5(1):48-56.

12. Sanches AM. Acurácia do preenchimento dos atestados de óbito com validação diagnóstica por meio das autopsias realizadas em Hospital Universitário do Município de São Paulo, 2001 [tese]. São Paulo: Universidade de São Paulo; 2003.

13. Alderson MR, Meade TW. Accuracy of diagnosis on death certificates compared with that in hospital records. Br J Prevent Soc Med. 1967;21(1):22-9.

14. Schwartz CJ. The Hawaii mortality followback study. II. An evaluation of medical certification of cause of death through the use of hospital discharge diagnoses. Honolulu: Hawaii State Department of Health; 1977. (R \& S Report, 17).

15. Gittelsohn A, Senning J. Studies on the reliability of vital and health records: 1 . Comparison of cause of death and hospital record diagnoses. Am J Public Health. 1979;69(7): 680-9.

16. Muñoz Rojas C. Calidad de la certificación y de la codificación de la causa de muerte, Estado de Falcón, 1986. Cuad Esc Salud Publica. 1987;49:57-74.

17. Pañella Noguera $H$, Thio $C B$, Rodríguez Sarmiento C, Roca Antonio J. [Certification of main cause of death in Barcelona, 1985]. Med Clin (Barc). 1989;92(4):129-34

18. Moussa MA, Shafie MZ, Khogali MM, elSayed AM, Sugathan TN, Cherian G, et al. Re- liability of death certificate diagnoses. J Clin Epidemiol. 1990;43(12):1285-95.

19. Laurenti R. Análise da mortalidade por causa básica e por causas múltiplas. Rev Saude Publica. 1974;8:421-35.

20. Laurenti R, Buchalla CM, de Lollio CA, Santo $\mathrm{AH}$, Jorge MHPM. Mortalidade de mulheres em idade fértil no município de São Paulo (Brasil), 1986, Mortes por Causas Maternas. Rev Saude Publica. 1990;24(6):468-72.

21. Carvalho ML, Niobey FML, Miranda NN e Sobroza PC. Concordância na determinação da causa básica do óbito em menores de um ano na região metropolitana do Rio de Janeiro. Rev Saude Publica. 1990;24(2):20-7.

22. Niobey FML, Cascão MM, Duchiale MP, Sobroza PC. Qualidade do preenchimento de atestados de óbito de menores de um ano na região metropolitana do Rio de Janeiro. Rev Saude Publica. 1990;24(4):311-8.

23. Nobre LC, Victora CG, Barros FC, Lombardi C, Teixeira AM, Fuchs SC. Avaliação da qualidade de informação sobre a causa básica de óbitos infantis no Rio Grande do Sul (Brasil). Rev Saude Publica. 1985;23(3):207-13.

24. Schnitman A. Análise da fidedignidade da declaração da causa básica de morte por câncer em Salvador, Brasil. Rev Saude Publica. 1990;24(6):490-6.

25. Monteiro GTR. Validação diagnóstica dos atestados de óbito com neoplasia como causa básica no estado do Rio de Janeiro [dissertação]. Rio de Janeiro: Escola Nacional de Saúde Pública, FIOCRUZ; 1995.

26. Puffer RR, Verhoestrate LJ. La mortalidad a causas de enfermedades cardiovasculares por países, con referencia especial a las enfermedades cardíacas arterioscleróticas. Revista Panam Salud Publica. 1958;44:474-83.

27. Puffer RR, Griffith GW. Características de la mortalidad urbana - Informe de la Investigación Interamericana de Mortalidad. Washington: PAHO; 1968. (Publicación científica $\left.n^{\circ} 151\right)$.

28. Puffer RR, Serrano CV. Características de la mortalidad en la niñez. Washington: OPS/ OMS; 1973. (Publicación científica ${ }^{\circ}$ 262).

29. Laurenti R, Mello Jorge MHP, Gotlieb SLD. A mortalidade materna nas capitais brasileiras: algumas características e estimativa de um fator de ajuste. Rev Bras Epidemiol. 2004;7(4) $449-60$.

30. Brasil, Ministério da Saúde. Estudo da mortalidade de mulheres de 10 a 49 anos, com ênfase na mortalidade materna. Brasília: Ministério da Saúde; 2006.

31. Kelson N, Farebrother M. The effect of inaccuracies in death certification and coding practices in the European Economic Community (EEC) on international cancer mortality statistics. Int J Epidemiol. 1987;16(3):411-4.

32. Percy C, Dolman A. Comparison of the coding of death certificates related to cancer in seven countries. Public Health Report. 1978; 93(4):325-50.

33. Centro Brasileiro de Classificação de Doenças. Avaliação entre codificadores. Boletim do Centro da OMS para a classificação de doenças em português. 1985;6:1-12.
34. Laurenti R. Causas múltiplas de morte [tese] São Paulo: Universidade de São Paulo; 1973.

35. Mello Jorge MHP. Situação atual das estatísticas oficiais relativas à mortalidade por causas externas. Rev Saude Publica. 1990;24(3): $317-23$.

36. Laurenti R, Mello Jorge MHP, Gotlieb SLD. O Sistema da informação sobre mortalidade: passado, presente e futuro. São Paulo: CBCD; 2006. (Série Divulgação ${ }^{\circ} 11$ )

37. Mello Jorge MHP, Cascão AM, Carvalho-Silva $R$. Acidentes e violências: um guia para a melhoria da qualidade da informação. São Paulo: CBCD; 2005. (Série Divulgação nº 10).

38. Almeida MTCGN; Beltrão KI, Leite IC. Vigilância da mortalidade materna: a identificação de mortes durante o ciclo gravídico puerperal ampliado. Cad Saude Colet (Rio J). 2006;14(2):265-82.

39. Organização Mundial da Saúde (OMS). Classificação estatística internacional de doenças e problemas relacionados à saúde. Vol. 2 e 3. 10a revisão. São Paulo: CBCD; 1995.

40. Moriyama IM. Public Health uses of mortality data: Em: Proceedings of the meeting on socioeconomic determinants and consequences of mortality. Cidade do México: United Nations; 1979. Pp. 8-25.

41. Pavillhon G, Chérié-Challine L. Randomised comparison of death certificates with and without guidelines. Em: Coleman M, Aylin P, eds. Death certification and mortality statistics: an international perspective. Studies on medical and population subjects $n^{\circ} 64$. Londres: National Statistics; 2000

42. Leitch DG, Heller RF, O'Connor SJ. Variation in death certification of ischaemic heart disease in Australia and New Zealand. Aust N Z J Med. 1987;17(3):309-15.

43. Kelson MC, Heller RF. The effect of death certification and coding practices in observed differences in respiratory diseases mortality in 8 E.E.C. countries. Rev Epidemiol Sante Publique. 1983;31(4):423-32.

44. Balkau B, Jougla E, Popoz L. European study of the certification and coding of causes of death of six clinical histories of diabetic patients. EURODIAB Subarea Study Group. Int J Epidemiol. 1993;22(1):116-26.

45. Aylin P. Revised medical certificate of cause of death in England: a pilot study. Em: Coleman MP, Aylin P, eds. Death certification and mortality statistics: an international perspective. Studies on medical and population subjects $n^{\circ}$ 64. Londres: National Statistics; 2000.

46. Laurenti R, Mello Jorge MHP. O atestado de óbito. São Paulo: CBCD; 2006. (Série Divulgação ${ }^{\circ} 1$; edição revista e aumentada).

47. Hill K. Making deaths count. Bull World Health Organ. 2006;84(3):162.

Manuscrito recebido em 21 de maio de 2007. Aceito em versão revisada em 27 de novembro de 2007. 
ABSTRACT Mortality statistics are used in epidemiology and public health as an indicator of health status, to evaluate health programs, and in population studies to compare trends and spatial differences. One of the variables used in this type of analysis is the

Underlying cause-of-death mortality statistics: considering the reliability of data underlying cause-of-death. However, the quality of cause-of-death statistics based on the information recorded by physicians in death certificates has been criticized. The aim of this paper is to discuss the reliability of cause-of-death data recorded by physicians in death certificates, based on studies carried out according to various methodologies, and to comment on the validity of using such underlying cause-ofdeath statistics.

Key words Mortality rate, statistical analysis, death certificates. 